\title{
Students' dynamic geometric reasoning about quantum spin-1/2 states
}

\author{
Hunter G. Close, Catherine C. Schiber, Eleanor W. Close, and David Donnelly
}

Department of Physics, Texas State University, San Marcos, TX 78666

\begin{abstract}
Quantum states are traditionally cognitively managed exclusively with algebra rather than geometry. One reason for emphasizing algebra is the high dimensionality of quantum mathematical systems; even spin-1/2 systems require a 2-d complex number space for describing their quantum states, which can be hard to visualize. Using "nested phasor diagrams," which use nesting to increase the dimensionality of graphic space, we taught undergraduate students to represent spin-1/2 states graphically as well as algebraically. In oral exams, students were asked to identify which spin-1/2 states, expressed arithmetically, would generate the same set of probabilities as each other (i.e., they are the same except for a different overall phase factor). Video records of oral exams $(\mathrm{N}=13)$ show that no student performed this task successfully using an algebraic method; instead, all successful students solved the problem graphically. Furthermore, most students who were successful used a certain gesture to solve the problem.
\end{abstract}

Keywords: graphics, spatial cognition, gesture, quantum mechanics, spin.

PACS: 01.40.Fk, 01.40.Ha, 03.65.Aa

\section{INTRODUCTION}

Quantum mechanics is regarded by most who encounter it as extremely abstract and difficult to understand. It is also traditionally presented mostly in algebraic form with expressions that are difficult to interpret in geometric or physical terms. Studies on student difficulties in quantum mechanics show overall that students are often at a loss to explain algebraic expressions conceptually or graphically $[1,2]$. Research on student understanding of spin [3] indicates that students have difficulty distinguishing between physical 3-d "laboratory" space and the 2-d complex Hilbert space of spin quantum states. The instructional tools and findings presented in this article may contribute to solving this problem by helping students build geometric intuition about spin.

This article is also part of a sustained research project investigating student learning of complex numbers and functions, both in terms of real and imaginary parts as well as in terms of phase. Previous research by others $[1,2,4]$ has shown that many students have difficulty correctly applying and interpreting imaginary parts and phases.

\section{BACKGROUND}

\section{Spin-1/2 systems}

Every spin-1/2 state can be expressed as $|\psi\rangle=c_{1}|+z\rangle+c_{2}|-z\rangle=R_{+} e^{i \theta_{+}}|+z\rangle+R_{-} e^{i \theta_{-}}|-z\rangle$ $=e^{i \theta_{+}}\left(R_{+}|+z\rangle+R_{-} e^{i\left(\theta_{-}-\theta_{+}\right)}|-z\rangle\right)$, where $|+z\rangle$ and $|-z\rangle$ represent a basis of spin-up and spin-down states along the $z$-axis. Probabilities for spin measurements along different directions in 3-d lab space generally depend only the magnitudes $R_{+}$and $R_{-}$(constrained via normalization to $R_{+}^{2}+R_{-}^{2}=1$ ) and the relative phase $\theta_{-}-\theta_{+}$, but never on the overall phase $\theta_{+}$.

\section{Nested Phasor Diagrams}

The nested phasor diagram (NPD) [5] is a graphic representation of a quantum spin-1/2 state (or any twostate quantum system) that uses nesting to display the four parameters $R_{+}, R_{-}, \theta_{+}$, and $\theta_{-}$, plus the orthogonality relation $\langle+z \mid-z\rangle=0$ and normalization, all on a 2-d surface. We designed the NPD on the basis of principles of graphic design for displaying quantitative information [6]. The NPD for an arbitrary state is shown in fig. 1, with explanatory labels.

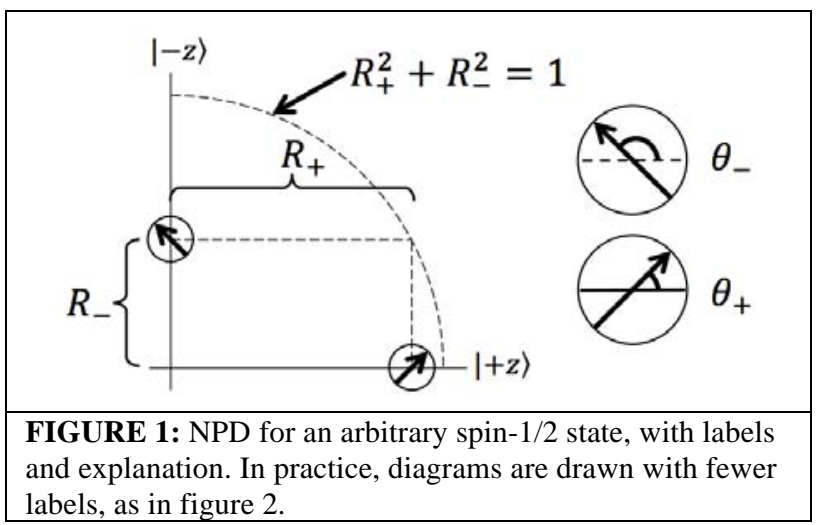




\section{DATA AND METHODS}

\section{Course context}

The data in this study are video records of the first of two oral examinations in an upper-division firstsemester course in quantum mechanics, taught by the first author. Students had previously taken a typical modern physics course that included an introduction to the time-independent Schrodinger equation, including finding wave functions for the infinite square well. The quantum mechanics course followed a "spin first" approach [7] that taught students about spin not as an extension of the fairly advanced topic of angular momentum, but as a realistic example of a two-state system, with opportunities for in-depth treatment of fundamental concepts like non-commuting observables, uncertainty, and complex phase. Instruction on spin-1/2 states included frequent student practice with NPDs.

\section{Oral Examination}

The oral exam occurred about $1 / 3$ of the way through the semester. Each student $(\mathrm{N}=13$, labeled $\mathrm{S} 1-$ S13) signed up for a one-hour time slot with the instructor over the course of a 3-day period. The exams were video-recorded, and live field notes were recorded. The following framework for exam questions was provided to the students one week in advance of the exam, while specific details were revealed only during the exam.

\begin{tabular}{|c|}
\hline Framework for oral exam \\
\hline $\begin{array}{l}\text { 1. Find an unknown spin- } 1 / 2 \text { state from probabilities } \\
\text { of spin measurements in the } x, y \text {, and } z \text { directions. }\end{array}$ \\
\hline $\begin{array}{l}\text { 2. For a set of quantum states, determine which would } \\
\text { generate the same set of probabilities for spin } \\
\text { measurements and which would generate different sets } \\
\text { of probabilities, without needing to perform direct } \\
\text { calculations of these probabilities. }\end{array}$ \\
\hline
\end{tabular}

Student solutions to problem 1 were almost exclusively algebraic and are not reported here. Student solutions to problem 2 are the subject of the present analysis. Though students were all given identical problems to solve, the interaction between instructor and student during the exam was primarily improvised dialogue rather than scripted protocol.

\section{Method of video analysis}

All 13 videos of student performance on problem 2 were analyzed according to the students' use of various semiotic fields for solution, namely: written algebra or arithmetic; drawn graphics, especially NPDs; and gesturing, especially in relation to the NPDs.

\section{The "Distinguishable states" problem}

Students were provided a sheet of paper with a problem statement and list of quantum states, as shown in Table 1. The list provided to the students included five additional, more challenging, states not shown here. In almost all cases, students did not complete their analysis of states A-E with sufficient remaining time to move on to the additional states. The instructor clarified the meaning of the problem for students whenever needed.

TABLE 1. The "Distinguishable states" problem, given to students in an oral exam.

Which of these quantum states would generate the same set of probabilities for spin-1/2 measurements (in any/all directions) as each other, and which would generate different sets of probabilities?

\begin{tabular}{|c|c|c|}
\hline$|\psi\rangle=c_{1}|+z\rangle+c_{2}|-z\rangle$ & $c_{1}$ & $c_{2}$ \\
\hline $\mathrm{A}$ & $\frac{1}{\sqrt{2}}$ & $\frac{1+i}{2}$ \\
\hline $\mathrm{B}$ & $\frac{1+i}{2}$ & $\frac{1}{\sqrt{2}}$ \\
\hline $\mathrm{C}$ & $\frac{1+i}{2}$ & $\frac{1+i}{2}$ \\
\hline $\mathrm{D}$ & $\frac{1}{\sqrt{2}}$ & $\frac{1}{\sqrt{2}}$ \\
\hline $\mathrm{E}$ & $\frac{i}{\sqrt{2}}$ & $\frac{1+i}{2}$ \\
\hline
\end{tabular}

All states A-E are composed with coefficients that differ only in phase; each coefficient shown has magnitude $\frac{1}{\sqrt{2}}$. Thus, solving the problem requires an assessment of the phase information of these states. Furthermore, since changing the overall phase does not have an experimentally observable effect, the task amounts to a determination of which states have the same relative phase as each other, and which have different relative phases, regardless of overall phase.

Figure 2 shows NPDs for states A, B, and E, which can be used to show that the relative phase of state $B$ $\left(-\frac{\pi}{4}\right)$ is the same as that of E but different from that of A $\left(+\frac{\pi}{4}\right)$. Thus, states B and E will generate the same set of probabilities as each other, while A would generate a different set. States $C$ and $D$ each have a relative phase of zero, so they would generate the same probabilities as each other. Students usually 


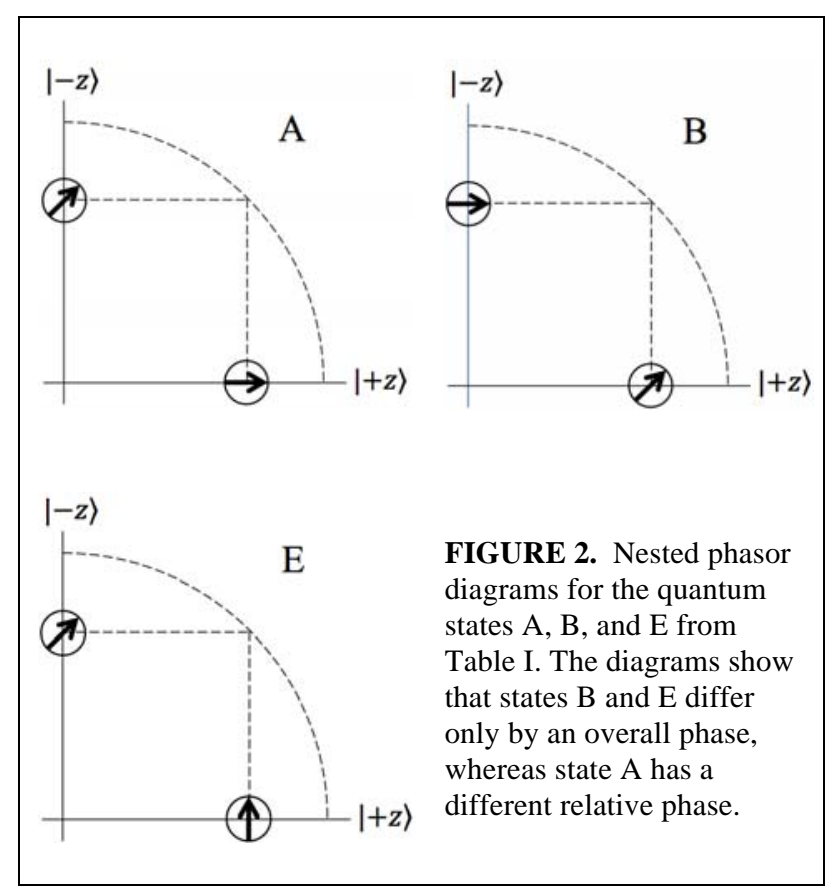

discovered that the "distinguishable states" problem had two natural parts, which we designate here as the $\mathrm{A} / \mathrm{B} / \mathrm{E}$ task and the $\mathrm{C} / \mathrm{D}$ task.

\section{RESULTS AND DISCUSSION}

\section{The "Constant Relative Phase" (CRP) gesture}

One gesture was particularly common (used at some point in the exam by 7 of 13 students) as students engaged the "distinguishable states" problem. We call this gesture the "constant relative phase" gesture (CRP) and describe it before other results, for reference. The prototypical performance of this gesture involves the student facing the whiteboard and simulating a NPD with the body, and using the body to animate a graduated transformation of one NPD on the board to another. The student presents both hands out in front of the body, closing all fingers except for the two index fingers, which are straight. Each finger represents the phase information of each component of the NPD. The fingers lie in a plane parallel to the whiteboard and are matched in orientation to those phase angles shown in the NPD. These fingers will in general be oriented to different angles. After matching the orientation of the fingers to the phase angles in one NPD, the fingers are rotated in a plane parallel to the board so as to maintain a constant difference in phase angle. The student rotates the fingers until one finger (usually that on the right hand) matches the phase angle of a second state for comparison, and checks to see if the other finger (on the left hand) matches the corresponding phase angle of the new state. If there is a match, the states have the same relative phase, and if not, the states have different relative phase. We invite the reader to try the gesture while examining fig. 2 .

\section{Quantitative trends in performance}

Frequent, coherent use of NPDs. Nine of 13 students spontaneously chose to draw NPDs during their interaction with the "distinguishable states" problem. The other 4 were prompted by the instructor to try using NPDs after they had tried unsuccessfully to approach the problem in different ways, usually after about 10 minutes. In all but one case (S6), students made substantive use of the NPDs they had drawn. In our prior theoretical work explaining the invention of NPDs, we claimed that nesting is "not inherently cognitively problematic," despite what might appear to be inconsistent meaning assigned to space in a graphic. We believe the empirical evidence provided here supports this claim.

Little gesturing for $C / D$. Eleven of 13 students were successful in recognizing that states $\mathrm{C}$ and $\mathrm{D}$ would generate the same probability sets. The other two students were either unsuccessful on this point (S6) or did not address it (S13). Of these 11, 7 students successfully compared states $\mathrm{C}$ and $\mathrm{D}$ with no gesture at all. The students who did gesture include one (S2) who briefly arranged his index fingers statically as though they were the phasors of state C. Three students (S3, S10, S12) gestured about C and D with a CRP gesture. We hypothesize that few students gestured on this task because it was easier for them to see plainly that, for each state, its two components had the same phase. Of the three who did perform the CRP gesture, two (S10, S12) were low-performers on the exam and appeared to have a low facility in general with complex numbers. Thus it may be that the gesture helped these weaker students on a task that for other students was too easy to require a helpful gesture.

Error managing nested aspect in $A / B / E$. Three students (S1, S2, S10) made the following error on the $\mathrm{A} / \mathrm{B} / \mathrm{E}$ task: They switched the phasors for the components of state E. This switch led them to conclude that $\mathrm{E}$ had the same relative phase as $\mathrm{A}$, not B. We hypothesize a reason for this particular error: The pure imaginary aspect of the coefficient $c_{1}$ for state E cued a sense of verticality that had an overriding effect on the student's otherwise correct knowledge of how to construct the diagram. Put simply, the $i$ seemed like it should go on the vertical axis. In terms of our theory of nesting, ${ }^{5}$ the students failed in this instance to reserve the idea " $i$ is vertical" for the region inside the phasor's "nest," and instead allowed the idea to apply outside the nest, where "vertical" goes with $c_{2}$. However, we do not believe this error should be described as a difficulty with or 
confusion about NPDs in general, since it was an isolated error made in the context of a more complicated task and therefore probably in haste.

Success on $A / B / E$ mediated by $C R P$ gesture. Nine students were essentially successful on the A/B/E task. This count includes $\mathrm{S} 1, \mathrm{~S} 2$, and $\mathrm{S} 10$ from the previous paragraph because their reasoning on the basis of their NPDs was correct. Of these nine, seven students performed the CRP gesture as an essential step in their successful recognition of which diagrams featured the same relative phase. Of the two students who were successful on the task without the CRP gesture, one (S1) made a lesser gesture, in which he waved one hand through the relative phase angle of one diagram, seeming to recognize it as matching the other diagram; and the other (S4) bridged his sense of the diagrams to his conclusion by naming the phase angles verbally: "45 degrees" or "negative 45 degrees." We wondered, considering that the CRP gesture is a two-handed operation, whether students (S1, S4) who held a marker or paper might be disinclined to perform it. This may be an inhibiting factor, but it's curious to observe that S10 performed CRP with a marker in one hand and a paper in the other, so if there is a rule that objects in hands inhibit gesturing, it is not absolute.

Static wedge gesture in $A / B / E$. Two students (S5, S7) made static "wedge" gestures, joining their wrists at a vertex and forming their hands into straight edges. This gesture seems related to a conceptual error each explained in words: "Relative phase is just the distance between the phases." These students stated incorrectly that $\mathrm{A}, \mathrm{B}$, and $\mathrm{E}$ all had the same relative phase. It seems that performing a deliberate moving gesture from one angular position to another corresponds to a correct "sign-sensitive" concept of relative phase, while the static gesture indicates an incorrect "absolute-value" concept. Thus, it seems that the "motion" is not just either present or not in the gesture, but also in the thinker's concept. This result is consistent with the foundation of conceptual metaphor theory [8], which says that metaphor is primarily not a surface, linguistic phenomenon, but a deep, conceptual one.

\section{IMPLICATIONS}

\section{Implications for instruction}

First we have found that students have shown their ability to make productive use of a graphic tool, the NPD, to represent two complex coefficients for spin$1 / 2$ quantum states, which are traditionally not conceived graphically or geometrically. The conventional wisdom is that such states, as mathematical entities composed of two complex numbers, are too abstract for such a representation. The general implication here is that increased geometrization of canonical physics content can be of some advantage to students. Second, we have shown that students made productive use of their bodies in cognitive interaction with the graphic. Instruction should provide as many opportunities as possible for students to make sense of ideas, including the opportunity for body movement, whether known from research like the present analysis or as yet undiscovered.

\section{Implications for research on cognition}

We find it to be especially curious that assessing relative phases by visual examination of the NPDs seems too difficult in the case of the $\mathrm{A} / \mathrm{B} / \mathrm{E}$ task, while performing a coordinated rotation of different fingers at different angles is apparently more manageable. Considering this fact together with students' facility with NPDs, in which some differences between quantum states are conceived as rotation, makes us wonder what fundamental importance rigid-body rotation might have in cognition; in terms of both enacted rotation as an aid to imagined rotation, and imagined rotation as a metaphorical aid for understanding change in general.

\section{REFERENCES}

1. C. Singh, "Student understanding of quantum mechanics at the beginning of graduate instruction" $\mathrm{Am}$. J. Phys. 76 (3), 277-287 (2008).

2. G. Zhu \& C. Singh, "Surveying students' understanding of quantum mechanics in one spatial dimension," Am. J. Phys. 80 (3), 252-259 (2012).

3. G. Zhu \& C. Singh, "Improving students' understanding of quantum mechanics via the Stern-Gerlach experiment" Am. J. Phys. 79 (5), 499-507 (2011).

4. S. B. McKagan et al., "Developing and researching PhET simulations for teaching quantum mechanics" Am. J. Phys. 76 (4\&5), 406-417 (2008).

5. H. G. Close, E. W. Close, and D. Donnelly, "Nesting in graphical representations in physics," in Physics Education Research Conference-2012, edited by P. Engelhardt, A. Churukian, and N. S. Rebello, AIP Conference Proceedings 1513, Melville, NY, 2013, pp. 110-113.

6. E. R. Tufte, Beautiful Evidence, Graphics Press, Cheshire, CT (2006).

7. D. H. McIntyre, Quantum Mechanics, Pearson, Boston (2012).

8. G. Lakoff \& M. Johnson, Philosophy in the flesh: The embodied mind and its challenge to Western thought, New York, Basic Books (1999). 